\title{
Der Trost der Worte
}

Interview mit Karin Schneuwly: Adrian Ritter

Freier Journalist

\author{
Dass Krankenhäuser im 19. Jahrhundert begannen, Bibliotheken für Patienten ein- \\ zurichten, ist kein Zufall: Literatur kann heilsam sein. Diese Erfahrung macht auch \\ Karin Schneuwly als Bibliotherapeutin.
}

\begin{abstract}
Karin Schneuwly, Sie helfen als Bibliotherapeutin mit Literatur anderen Menschen. Gibt es ein Buch, das Ihnen persönlich speziell geholfen hat?

Ich war vor vielen Jahren in einen Mann verliebt, die Liebe war obsessiv und hat mich bei der Arbeit gebremst. Ich habe nur noch auf einen Anruf von ihm gewartet. Da hat mir ein Freund «Des Menschen Hörigkeit» von Somerset Maugham empfohlen. Darin habe ich mich gespiegelt gefunden, ich habe gesehen, dass ich nicht alleine bin mit diesem Problem. Das hat mir geholfen, loszulassen. Ich habe auch gemerkt, dass ich während der Dauer des Lesens des dicken Buches an etwas anderes dachte - das war eine gute Erfahrung.
\end{abstract}

Hat das zur Idee geführt, Bibliotherapeutin zu werden? Diese Erfahrung ist mir natürlich in Erinnerung geblieben. Zuerst habe ich aber als Co-Programmleiterin viele Jahre im Literaturhaus Zürich gearbeitet. Dabei habe ich erlebt, wie Menschen sich anhand von Literatur öffnen und ihr Leben erzählen wollen. Texte bringen etwas in Gang, das hat mich fasziniert. Das hat mich dazu bewogen, nach der Ausbildung zur Kunsttherapeutin noch eine Ausbildung in Bibliotherapie zu absolvieren.

\section{Wer kommt heute zu Ihnen in die Bibliotherapie?}

Es sind einerseits Menschen, die sich in einer Krise befinden, vielleicht eine Psychotherapie besuchen und begleitend fokussiert etwas lesen oder aufschreiben möchten. Andererseits sind es auch einfach neugierige Menschen, die mehr über sich selber erfahren möchten. Eines ist ihnen gemeinsam: Sie schreiben und lesen gerne oder haben das in der Kindheit getan und den Zugang etwas verloren.

\section{Wie kann Bibliotherapie diesen Menschen helfen?}

Bibliotherapie nutzt die heilsame Kraft der Sprache und der Geschichten. Literatur gibt einem die Möglichkeit, eine andere Perspektive kennenzulernen. Wenn ein Text einen packt, kommt man in einen «Flow», wie Psychologen das nennen. Ein Ratgeberbuch wird so etwas nicht schaffen. Sachliteratur vermittelt Wissen

\section{Zur Person \\ Karin Schneuwly hat Germanistik, Philosophie und Vergleichende Literaturwissenschaft studiert und arbeitete danach im Unionsverlag in Zürich und als Lektorin bei Lars Müller Publishers. Anschliessend war sie während neun Jahren für das Programm und Projekte des Li- teraturhauses Zürich verantwortlich. Heute ist sie selbstständige Texte- rin, Lektorin und Bibiliotherapeu- tin. Sie hat nach einer Ausbildung in Kunsttherapie noch eine Ausbildung in Bibliotherapie am Fritz-Perls-Institut absolviert. www.text-praxis.ch}

und vernachlässigt dabei das Erleben des Einzelnen. In einem literarischen Werk hingegen wird Vergangenheit, Gegenwart und Zukunft gleichzeitig erfahrbar, und das ist wichtig.

Zudem spendet Literatur Trost. Das merkt man schon mit kleinen Kindern, die ein blutendes Knie haben: Die Mutter singt ein Lied oder erzählt eine Geschichte, und der Schmerz ist vergessen. Das hilft oft auch bei Erwachsenen: Man nimmt sich Zeit für sich und kann seine Leiden mittels Literatur spiegeln. Beim Lesen macht man etwas durch, ohne dass einem dabei etwas passiert. Das stärkt auch die Aufmerksamkeit. Insofern ist Bibliotherapie auch eine Form der Prävention, indem man nützliche Eigenschaften schult und sich selber besser kennenlernt.

\section{Wird in der Bibliotherapie vor allem gelesen und darüber gesprochen oder auch geschrieben?}

Manchmal gebe ich meinen Klienten eine Liste von Büchern, die sie zu Hause lesen und die wir einzeln diskutieren. Das können ganz kurze Texte sein - eine Erinnerung oder ein kleines Gedicht. Wir kommen über das Lesen oder Vorlesen immer auch zum selber Schreiben. Als Kunsttherapeutin nutze ich eine ganze Palette an Zugängen, je nach Situation. Manchmal zeichnen wir zuerst in einer Sitzung und kommen über das Zeichnen zum Schreiben. 
Gibt es Studien zur Wirksamkeit der Bibliotherapie?

Ja, es gibt zum Beispiel Studien, die zeigen, dass Kinder ihre Wahrnehmung und Handlungsfähigkeit erweitern können, wenn man ihnen eine Geschichte erzählt. Das geschieht meiner Erfahrung nach auch bei Erwachsenen, die selber lesen. Ich erhalte positive Rückmeldungen von meinen Klienten. Sie erzählen mir etwa, dass sie sich mehr Zeit nehmen, um mit Büchern nach der Arbeit zur Ruhe zu kommen. Klienten, die Kinder haben, sagen mir, dass sie viel gezielter Bücher aussuchen, um gegen Ängste ihrer Kinder anzukämpfen.

Hilft Bibliotherapie auch bei somatischen Beschwerden? Es gibt Studien aus Grossbritannien, die zeigen, dass Patienten mithilfe eines Lesezirkels ihre Medikamente reduzieren konnten. Ich habe früher in einem Spital eine Bibliotherapie-Gruppe für Schmerzpatienten angeboten. Die Rückmeldung der Teilnehmenden war, dass sie ihre Schmerzen während der Stunde des Lesens und Schreibens vergessen konnten. Die Patienten lernen auch, mithilfe von Literatur Worte für ihren Schmerz zu finden. Vor allem aber geschieht in der Bibliotherapie eine Ablenkung, weg vom Schmerz. Deshalb begannen Krankenhäuser im 19. Jahrhundert, Bibliotheken einzurichten: Lesende Patienten sind die ruhigeren und optimistischeren Patienten.

\section{Das waren die Anfänge der Bibliotherapie?}

Das war der Anfang der gezielten Nutzung der Bibliotherapie. Ihre Quelle ist schon in der Antike zu finden, wo Heilung und Literatur zusammengehörten. Apollon war sowohl der Gott der Dichtkunst als auch der Heilkunst. Auch Aristoteles vertrat in seiner Katharsislehre die Idee, dass eine Tragödie - also ein Schauspiel - die Zuschauer in die dargestellte Handlung hineinversetzen soll. Dadurch würden Ergriffenheit und Mitgefühl entstehen, was beim Zuschauer eine Reinigung Katharsis - bewirke.

\section{Gibt es Situationen, in denen Bibliotherapie nicht sinnvoll ist?}

Bibliotherapie arbeitet ressourcenorientiert, spielerisch, sie kann daher eigentlich keinen Schaden anrichten. Aber es braucht Lust darauf: Lesen und Schreiben ist auch Arbeit, etwas Aktives. Menschen in einer Depression ha-

\section{Buchhinweise}

Karin Schneuwly: Glück besteht aus Buchstaben. Nagel \& Kimche, Zürich, 2017 Ella Berthoud, Susan Elderkin, Trudl Bünger: Die Romantherapie - 253 Bücher für ein besseres Leben, Insel Taschenbuch, 2014

Hilarion G. Petzold, Brigitte Leeser, Elisabeth Klempnauer (Hrsg.):Wenn Sprache heilt. Handbuch für Poesie- und Bibliotherapie, Biographiearbeit und Kreatives Schreiben. Festschrift für Ilse Orth, Aisthesis Verlag, 2017

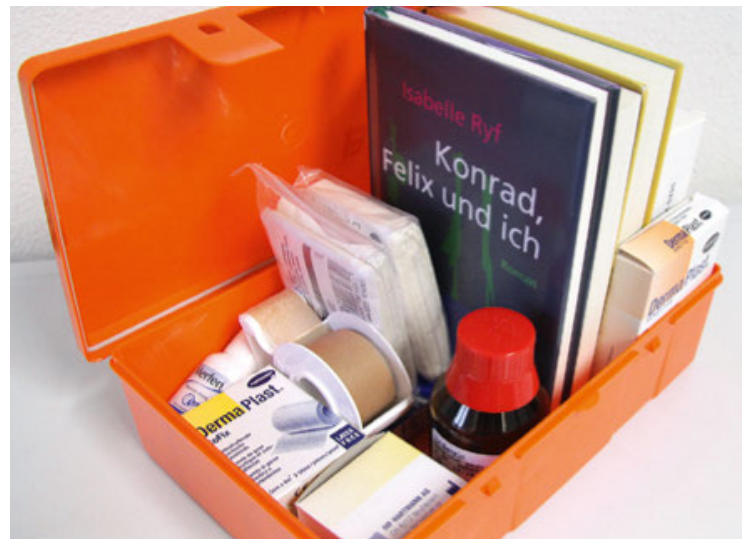

Ein gutes Buch in der Hausapotheke kann nicht schaden: Auch Literatur kann heilen.

ben meist Mühe, sich auf ein Buch zu konzentrieren. Hier bietet es sich eher an, mit Gedichten zu arbeiten - durchaus auch Gedichte, die einen dunklen, schweren Inhalt haben. Ganz wichtig ist das Gespräch über das Gelesene.

\section{Wie finden Sie für eine bestimmte Person das geeig- nete Buch?}

Grundsätzlich gehe ich nach dem Lust-Prinzip vor: Ich liebe ein Buch, das mir für die Situation eines Klienten passend scheint und versuche die Person dafür zu begeistern, es zu lesen. Manchmal lese ich auch ein Gedicht vor und suche nach einer Resonanz. Ich frage die Klienten immer auch, was sie selber schon gelesen haben. Ich habe selber viel gelesen und diese Bücher nach Motiven und Themen systematisiert. Zudem gibt es das Buch «Romantherapie» von Ella Berthoud und Susan Elderkin. Darin sind 253 Bücher nach ihrer Wirkung aufgelistet.

\section{Gibt es Bücher, die Sie immer wieder empfehlen?}

"Wer die Nachtigall stört» von Harper Lee habe ich schon mehrfach empfohlen. Es geht darin um einen alleinerziehenden Mann, der Verantwortung für seine Kinder übernimmt. Der Roman behandelt viele Themen wie Kindheit, Gerechtigkeit und Sinn. Nicht zuletzt ist der Vater eine vorbildhafte Erwachsenenperson.

\section{Wie sieht es mit der Nachfrage nach Bibliotherapie aus?} Schreibwerkstätten etwa in Altersheimen oder Schulen werden häufiger angeboten als früher, das scheint mir ein klarer Trend zu sein. In Deutschland wird Bibliotherapie bisweilen sogar in Gefängnissen angeboten. Im psychiatrischen Kontext sind andere Kreativtherapien wie Mal- oder Musiktherapie im deutschsprachigen Raum weiter verbreitet als die Bibliotherapie. Das ist in den USA und in skandinavischen Ländern anders. Dort wird Poetry Therapy sogar an Universitäten gelehrt.

Bildnachweis

Koffer: Adrian Ritter; Porträtfoto: zVg 